\title{
From macrophage biology to macrophage-based cellular immunotherapies
}

\author{
Elvira Mass $\mathbb{B}^{1} \cdot$ Nico Lachmann $\mathbb{1}^{2,3,4}$
}

Received: 25 June 2020 / Revised: 24 September 2020 / Accepted: 27 October 2020 / Published online: 4 February 2021

(c) The Author(s) 2021. This article is published with open access

\section{The concept of macrophages}

As far back as 1882, Ilya (Elie) Metchnikoff was the first to discover macrophages (MФ) and their important role in host defense [1]. Now, almost 140 years later, $\mathrm{M} \Phi$ are known as incredible cell types with unique functions and capabilities. In fact, Ilya Metchnikoff paved our understanding of peripheral blood monocytes and $\mathrm{M} \Phi$ in the clearance of pathogens, while follow-up studies described $M \Phi$ in various organs in which their defined functions are directly linked to a divergent onset (Fig. 1).

$\mathrm{M} \Phi$ are found in all organs, typically forming a threedimensional network that supports tissue function by production of growth factors and phagocytosis of dead and dying cells during steady state. Moreover, MФ are among the first cells encountering pathogens, debris, or tumor cells in diseased tissue. Given these and other multifarious functions and their important role in the onset and/or progression of various diseases, $\mathrm{M} \Phi$ are therefore rendered as a perfect candidate in cell-based immunotherapies.

Against a great body of newly emerging data, scientists still encounter in the MФ research literature the terminology of $\mathrm{M} \Phi$ polarization, most commonly into the M1 (classically activated) and M2 (alternatively activated) phenotypes (see also review [2]). Thus, also therapy approaches aim at

Nico Lachmann

Lachmann.nico@mh-hannover.de

1 Developmental Biology of the Immune System, Life \& Medical Sciences (LIMES) Institute, University of Bonn, Bonn, Germany

2 Translational Hematology of Congenital Diseases, Institute of Experimental Hematology, Hannover Medical School, Hannover, Germany

3 REBIRTH Research Center for Translational and Regenerative Medicine, Hannover, Germany

4 Department for Pediatric Pulmonology, Allergology and Neonatology, Hannover Medical School, Hannover, Germany using this apparent plasticity of $\mathrm{M} \Phi$ and "simply" try to switch them from M1 to M2 or the other way around, depending on whether anti- or pro-inflammatory responses are required. However, these M1/M2 definitions stem from cells treated with interferon- $\gamma$ or interleukin- 4 , respectively, and ignore the fact that these defined and isolated stimuli in vitro do not emerge in vivo. In contrast, under physiological conditions a plethora of stimuli (e.g., cytokines, bacteria, foreign antigens, or the tissue niche) modulate $M \Phi$ function in an orchestrated fashion, which can be difficult to recapitulate in vitro [2].

\section{Macrophage ontogeny $=$ macrophage function?}

Given the demonstration in recent years that tissue-resident and recruited MФ represent different developmental lineages, there is growing evidence for their nonredundant functions during steady state and disease. While most tissues harbor only a minor fraction of monocyte-derived $M \Phi$, the majority of tissue-resident $\mathrm{M} \Phi$ originate from erythromyeloid progenitors (EMPs) in the yolk sac [3] that are maintained throughout life largely independent of the circulating hematopoietic system by local proliferation. Thus, the observed plasticity and multi-functionality of $M \Phi$ in healthy and diseased tissues may not only be triggered by environmental cues, but also already determined by $М \Phi$ ontogeny. One such example is the liver: EMP-derived Kupffer cells emerge early during embryogenesis and are involved in hepatocyte metabolism and erythrocyte development and recycling - just to name a few of many functions-while monocyte-derived liver capsular $M \Phi$ sense and control entrance of peritoneal bacteria via recruitment of neutrophils [4].

That origin matters for M $\Phi$ identity and function has been shown in studies in which an empty $M \Phi$ niche was replenished by transplanted $\mathrm{M} \Phi$ progenitors of fetal or adult origin or by already differentiated $\mathrm{M} \Phi$ from other tissues. 


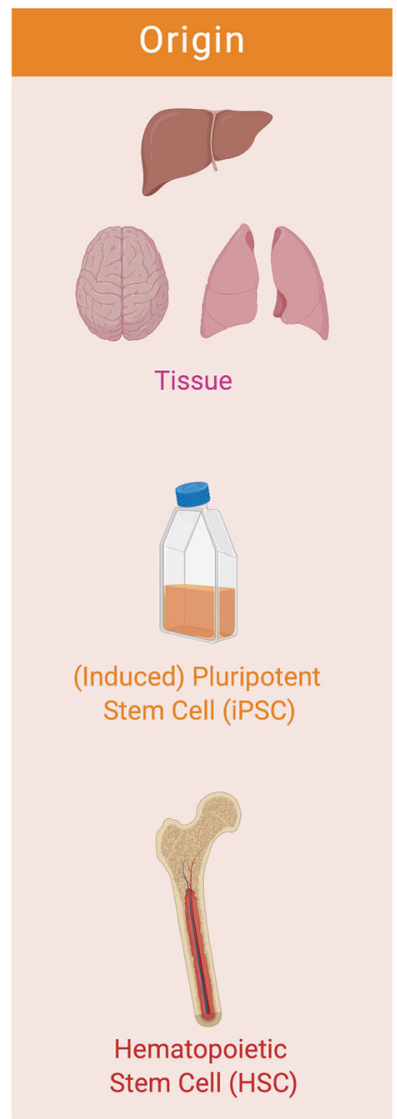

Fig. 1 Subsets of macrophages for adoptive cell therapies. Macrophages may be isolated or generated from tissues, induced pluripotent stem cells (iPSCs), or hematopoietic stem cells (HSCs). In fact, the origin and the starting cell material for the derivation of macrophages should be evaluated prior to generation. While the iPSC technology would allow for scalable and continuous generation of predominantly

While this concept would be applicable for, e.g., congenital diseases (e.g., CSF2RA/B deficiency, see below), other adoptive transfer scenarios would rely on the depletion of the endogenous $M \Phi$ pool to allow homing of cells. Clodronate liposomes, CSF1R antagonists, or sophisticated irradiation schedules may be applied alone or in combination to sufficiently deplete resident $M \Phi$ and/or infiltrating endogenous monocytes, while carefully evaluating potential off-target effects.

Most progenitors and fetal $\mathrm{M} \Phi$ are highly adaptive to their environment and establish a microglia-like phenotype in the brain or alveolar M $\Phi$ (AM) phenotype in the lung, and may even take over certain functions of the original $M \Phi$ population. Yet, in some cases $M \Phi$ of inappropriate origin show a distinct transcriptional profile and express disease-associated genes [5], or display decreased function during steady state and fail to protect from infection [6].

However, a generalized oversimplification of EMPderived $M \Phi$ being pro-regenerative and anti-inflammatory, while monocyte-derived $\mathrm{M} \Phi$ are rather pro-inflammatory, primitive macrophages, generation of HSC- or peripheral blood derived monocyte-derived macrophages would allow only for a single derivation of definitive cells. Following an, e.g., macrophage enhancement and/or purification step, generated macrophages may be infused into the patient to improve disease symptoms.

does not always apply. Depending on the molecular makeup of the cell or disease progression, EMP-derived $М \Phi$ can become pro-inflammatory and drivers of pathophysiology. Mutated EMP-derived microglia can cause neurodegeneration [7], and Kupffer cells experiencing a nonalcoholic steatohepatitis diet for 30 weeks lose their identity and transit toward a pathogenic phenotype similar to monocytederived $M \Phi[8]$.

$M \Phi$-based therapies should therefore consider the distinct ontogeny and the potential of $M \Phi$ and $M \Phi$ progenitors to adapt to (one of) these cells. Here, the developmental dichotomy could be represented by induced pluripotent stem cells (iPSCs)- or monocyte-derived M $\Phi$, respectively: while iPSC-derived M $\Phi$ (iPSC-M $\Phi$ ) may be easily harvested and collected for transplantation to mimic EMP-

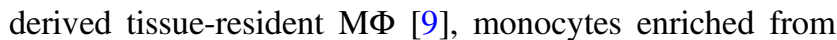
the blood of patients would differentiate into proinflammatory $\mathrm{M \Phi}$. In the future, it may be useful to employ both strategies simultaneously or even to combine this cell-therapy approach with a genetic therapy where one 
or both macrophage lineages are genetically corrected or modulated to treat diseases since the correct balance of M $\Phi$ of distinct origins is what every tissue needs for its homeostasis.

\section{(Pre)Clinical attempts of macrophage-based therapies}

As one attractive clinical roadmap for a $M \Phi$ therapy, liver cirrhosis has been introduced by the team of Stuart Forbes, using peripheral blood monocyte-derived MФ [10]. In a phase I clinical trial (ISRCTN 10368050), the authors isolated monocytes from individuals and infused M-CSFdifferentiated $\mathrm{M} \Phi$ intravenously back into the patients [11]. Designed as a dose-escalation study, the authors could not observe any reaction to the transfusion, neither doselimiting toxicity nor any sign of macrophage activation syndrome. While no adverse events could be monitored, overall efficacy still needs to be shown in patients. A similar liver-directed approach using $\mathrm{M} \Phi$ has been applied in the context of the rare disease heme oxygenase-1 (HMOX1) deficiency. In a preclinical study, the infusion of WT MФ into Hmoxl-deficient mice could proof engraftment and proliferation of cells and a correction of the disease phenotype [12]. To expand the field of $М \Phi$-based therapies, adoptive transfer of $\mathrm{M} \Phi$ directly into the lungs has been also recently highlighted for various diseases. In the context of Csf2r-deficient pulmonary alveolar proteinosis (PAP), first studies concentrated on bone marrow (BM)-derived $M \Phi$, which have been transferred as a single dose directly into different mouse models, which faithfully recapitulate the clinical phenotype of PAP [13, 14]. Given the longevity and the adaption of transferred BM-M $\Phi$ toward an AM phenotype, current attempts are underway to establish an autologous M $\Phi$-cell product, which is genetically corrected and ready to be transferred into CSF2RA-deficient patients. Similar to the approach of BM-derived M could also be shown therapeutically effective following intrapulmonary transfer into mouse models of PAP or severe combined immunodeficiency. Moreover, the new technique also paved the way for the exploration of iPSC$M \Phi$ in the context of infectious diseases, in which iPSCM $\Phi$ have been used as an antibiotic independent immunotherapeutic approach targeting bacterial infections of the lung and other organs. Beyond these studies, populations of MФ have been also introduced in the brain (microglia) or bone (osteoclasts) and the impact of these two МФpopulations in the onset and progression of different diseases has been highlighted. For the latter, a recent study could not only proof the onset of osteoclasts from embryonic EMPs, but could also demonstrate that transfusion of monocytic cells can rescue mice from an adult-onset osteopetrotic phenotype [15]. While microglia also emerge from an early EMP during embryogenesis, human iPSCMФ have been shown to functionally integrate into xenografted mouse brain and retain human microglia identity [16]. This observation may extend recent attempts, which directly transfer hematopoietic progenitors directly into the brain. Given the various attempts to adoptively transfer M $\Phi$ into various tissues, clearly the fields of organ transplantation and cancer immunotherapy have been targeting MФ directly or indirectly. While the onset of tumor-associated macrophages is diverse, redirecting $M \Phi$ to specifically recognize the tumor by chimeric antigen receptor (CAR) technology has been introduced as a completely new field in M $\Phi$-directed therapies. Redirecting $M \Phi$ either by the forced expression of an anti-CD19 or anti-HER2 CAR against leukemia or solid tumors, respectively, and the introduction of an intracellular phagocytosis signaling cascade could impressively demonstrate the future potential of these M $\Phi$ $[17,18]$. Of note, $\mathrm{M} \Phi$ have been further manipulated by, e.g., cell intrinsic or extrinsic modifiers and used in different applications such as cancer immunotherapy, renal transplantation, type 1 diabetes, and others [19-21].

Clearly, the seminal potential and function of $\mathrm{M} \Phi$ and the recent possibilities to generate $M \Phi$ in a scalable bioreactor differentiation system pave the way for innovative immunotherapies (Fig. 1). Besides the costly manufacturing, it is crucial to properly evaluate safety-related issues of either autologous or allogenic MФ in appropriate (pre) clinical studies in order to promote the use of these cells in the clinic to benefit a greater number of patients. Taken together, recent and future insights into the biology and onset of $M \Phi$ could further extend our understanding on the therapeutic use of these cells in a variety of different diseases and medical indications (Fig. 1).

Acknowledgements NL is supported by the Ministry (MWK) of Lower Saxony (Förderung aus Mitteln des Niedersächsischen Vorab) and by the European Research Council (ERC) under the European Union's Horizon 2020 research and innovation program (Grant Agreement No. 852178). EM is supported by the Deutsche Forschungsgemeinschaft (DFG, German Research Foundation) under Germany's Excellence Strategy_EXC 2151-390873048 and by the European Research Council (ERC) under the European Union's Horizon 2020 research and innovation program (Grant Agreement No. 851257). Figure 1 was created with Biorender online software.

Funding Open Access funding enabled and organized by Projekt DEAL.

\section{Compliance with ethical standards}

Conflict of interest The authors declare that they have no conflict of interest.

Publisher's note Springer Nature remains neutral with regard to jurisdictional claims in published maps and institutional affiliations. 
Open Access This article is licensed under a Creative Commons Attribution 4.0 International License, which permits use, sharing, adaptation, distribution and reproduction in any medium or format, as long as you give appropriate credit to the original author(s) and the source, provide a link to the Creative Commons license, and indicate if changes were made. The images or other third party material in this article are included in the article's Creative Commons license, unless indicated otherwise in a credit line to the material. If material is not included in the article's Creative Commons license and your intended use is not permitted by statutory regulation or exceeds the permitted use, you will need to obtain permission directly from the copyright holder. To view a copy of this license, visit http://creativecommons. org/licenses/by/4.0/.

\section{References}

1. Gordon S. Elie Metchnikoff: father of natural immunity. Eur J Immunol. 2008;38:3257-64.

2. Orecchioni M, Ghosheh Y, Pramod AB, Ley K. Macrophage polarization: different gene signatures in M1(LPS+) vs. classically and M2(LPS-) vs. alternatively activated macrophages. Front Immunol. 2019;10:1084.

3. Gomez Perdiguero E, Klapproth K, Schulz C, Busch K, Azzoni E, Crozet L, et al. Tissue-resident macrophages originate from yolksac-derived erythro-myeloid progenitors. Nature. 2015;518:547-51.

4. Sierro F, Evrard M, Rizzetto S, Melino M, Mitchell AJ, Florido $\mathrm{M}$, et al. A liver capsular network of monocyte-derived macrophages restricts hepatic dissemination of intraperitoneal bacteria by neutrophil recruitment. Immunity. 2017;47:374-88.e6.

5. Bennett FC, Bennett ML, Yaqoob F, Mulinyawe SB, Grant GA, Hayden Gephart M, et al. A combination of ontogeny and CNS environment establishes microglial identity. Neuron. 2018;98: 1170-83.e8.

6. Li F, Okreglicka KM, Pohlmeier LM, Schneider C, Kopf M. Fetal monocytes possess increased metabolic capacity and replace primitive macrophages in tissue macrophage development. EMBO J. 2020;39:e103205.

7. Mass E, Jacome-Galarza CE, Blank T, Lazarov T, Durham BH, Ozkaya $\mathrm{N}$, et al. A somatic mutation in erythro-myeloid progenitors causes neurodegenerative disease. Nature. 2017;549:389-93.

8. Seidman JS, Troutman TD, Sakai M, Gola A, Spann NJ, Bennett $\mathrm{H}$, et al. Niche-specific reprogramming of epigenetic landscapes drives myeloid cell diversity in nonalcoholic steatohepatitis. Immunity. 2020;52:1057-74.e7.

9. Ackermann M, Haake K, Kempf H, Kaschutnig P, Weiss AC, Nguyen AHH, et al. A 3D iPSC-differentiation model identifies interleukin-3 as a regulator of early human hematopoietic specification. Haematologica. 2020: https://doi.org/10.3324/haematol. 2019.228064.

10. Thomas JA, Pope C, Wojtacha D, Robson AJ, Gordon-Walker TT, Hartland S, et al. Macrophage therapy for murine liver fibrosis recruits host effector cells improving fibrosis, regeneration, and function. Hepatology. 2011;53:2003-15.

11. Moroni F, Dwyer BJ, Graham C, Pass C, Bailey L, Ritchie L, et al. Safety profile of autologous macrophage therapy for liver cirrhosis. Nat Med. 2019;25:1560-5.

12. Kim KS, Zhang DL, Kovtunovych G, Ghosh MC, Ollivierre H, Eckhaus MA, et al. Infused wild-type macrophages reside and self-renew in the liver to rescue the hemolysis and anemia of Hmox1-deficient mice. Blood Adv. 2018;2: 2732-43.

13. Suzuki T, Arumugam P, Sakagami T, Lachmann N, Chalk C, Sallese A, et al. Pulmonary macrophage transplantation therapy. Nature. 2014;514:450-4.

14. Happle C, Lachmann N, Skuljec J, Wetzke M, Ackermann M, Brennig $S$, et al. Pulmonary transplantation of macrophage progenitors as effective and long-lasting therapy for hereditary pulmonary alveolar proteinosis. Sci Transl Med. 2014;6: $250 \mathrm{ra} 113$

15. Jacome-Galarza CE, Percin GI, Muller JT, Mass E, Lazarov T, Eitler J, et al. Developmental origin, functional maintenance and genetic rescue of osteoclasts. Nature. 2019;568:541-5.

16. Xu R, Li X, Boreland AJ, Posyton A, Kwan K, Hart RP, et al. Human iPSC-derived mature microglia retain their identity and functionally integrate in the chimeric mouse brain. Nat Commun. 2020;11:1577.

17. Klichinsky M, Ruella M, Shestova O, Lu XM, Best A, Zeeman M, et al. Human chimeric antigen receptor macrophages for cancer immunotherapy. Nat Biotechnol. 2020;38:947-53.

18. Morrissey MA, Williamson AP, Steinbach AM, Roberts EW, Kern N, Headley MB, et al. Chimeric antigen receptors that trigger phagocytosis. eLife 2018;7:e36688. https://doi.org/10. 7554/eLife.36688.

19. Hutchinson JA, Riquelme P, Sawitzki B, Tomiuk S, Miqueu P, Zuhayra $\mathrm{M}$, et al. Cutting Edge: Immunological consequences and trafficking of human regulatory macrophages administered to renal transplant recipients. J Immunol. 2011;187:2072-8.

20. Shields CWT, Evans MA, Wang LL, Baugh N, Iyer S, Wu D, et al. Cellular backpacks for macrophage immunotherapy. Sci Adv. 2020;6:eaaz6579.

21. Parsa R, Andresen P, Gillett A, Mia S, Zhang XM, Mayans S, et al. Adoptive transfer of immunomodulatory M2 macrophages prevents type 1 diabetes in NOD mice. Diabetes. 2012;61: 2881-92. 